\title{
Temporal evolution of the pulse width in GRBs
}

\author{
E. Ramirez-Ruiz ${ }^{1,2}$ and E.E. Fenimore ${ }^{2}$ \\ 1 Facultad de Ciencias, Universidad Nacional Autónoma de México, Distrito Federal, 04510, Mexico \\ ${ }^{2}$ Los Alamos National Laboratory, Mail Stop D436, Los Alamos, NM 87545, U.S.A.
}

Received December 18, 1998; accepted March 23, 1999

\begin{abstract}
Many cosmological models of GRBs envision the energy source to be a cataclysmic stellar event leading to a relativistically expanding fireball. Particles are thought to be accelerated at shocks and produce nonthermal radiation. The highly variable temporal structure observed in most GRBs has significantly constrained models. By using different methods of statistical analysis in the time domain we show that the width of the pulses in GRBs time histories remain remarkably constant throughout the classic GRB phase. If the emission sites lie on a relativistically expanding shell, we determine both the amount of deceleration and the angular spread of the emitting region from the time dependency of the pulse width. We find no deceleration over at least $2 / 3$ of the burst duration and angular spreads of the complete emitting shell that are substancially smaller than $\Gamma^{-1}$. The lack of temporal evolution of the pulse width should be explained by any fireball shock scenario.
\end{abstract}

Key words: gamma-ray: bursts

\section{Introduction}

The cosmological origin of GRBs, established as a result of optical follow-up observations of fading X-ray counterparts to GRBs, requires an extraordinarily large amount of energy to flood the entire universe with gamma rays. The lack of apparent photon-photon attenuation of high energy photons implies substantial bulk relativistic motion. The bulk Lorentz factor, $\Gamma=\left(1-\beta^{2}\right)^{-1 / 2}$, must be on the order of $10^{2}$ to $10^{3}$. Two major scenarios involving relativistic shells have been developed. In the external shock models, a relativistic shell, that expands outward for a long period of time, is generated by a single release of energy during the merger. The shell coasts in a gammaray quiet phase for a certain period. Eventually, the shell becomes gamma-ray active due to the interactions with the external medium. If the shell has a velocity, $v=\beta c$, then the photons emitted on axis over a period $t^{\prime}$ ("proper time" in the comoving frame of the shell) arrive at a detector over a much shorter period, $T=\frac{t^{\prime}}{2 \Gamma}$. The duration of the event is set by the expansion of the shell and the complex temporal structure is due to inhomogeneities in the shell and/or the ambient material. The alternative theory is that a central site releases energy in the form of a wind or multiple shells over a period of time commensurate with the observed duration of GRB. Each subpeak in the GRB is the result of a separate explosive event in the central site. If the emission sites do indeed lie on a relativistically expanding shell, the pulse width in the time GRBs histories scales as $\Delta T=\Lambda \Delta t^{\prime}$, where $\Lambda$ is the Doppler factor, $\Lambda=\Gamma(1-\beta \cos \theta)$. Here $\theta$ is the angle of the motion of the emitting region with respect to the direction of the emission. In this paper, we proposed to determine the angular spread of the emitting region and the amount of deceleration from the time histories of many GRBs.

\section{Pulse width evolution obtained from time histories}

A visual inspection of the BATSE catalog of multiplepeaked time histories reveals that peaks usually have about the same duration at the beginning of the burst as near the end of the burst. Our aim is to characterize and measure the pulse shape as a function of arrival time. The aligned peak method measures the average pulse temporal structure, each burst contributes to the average by aligning the largest peak (Mitrofanov 1997). We used all 53 bursts from the BATSE 4B Catalog that were longer than $20 \mathrm{~s}$ and brighter than 5 photons $\mathrm{s}^{-1} \mathrm{~cm}^{-2}$. Each burst must have at least one peak, as determined by a peak-finding algorithm (similar to Li \& Fenimore 1996), in each third of its duration. The largest peak in each third was normalized to unity and a shifted in time, bringing the largest peaks of all bursts into common alignment. This method was applied in each third of the duration of the bursts. Thus, we obtained one curve of the averaged pulse shape for each different section of the bursts (as shown in Fig. 1). The average profile is notably identical in each $1 / 3$ of $T_{90}$ (we estimate the differential spread, $S$, to be $\sim 1 \%)$. 


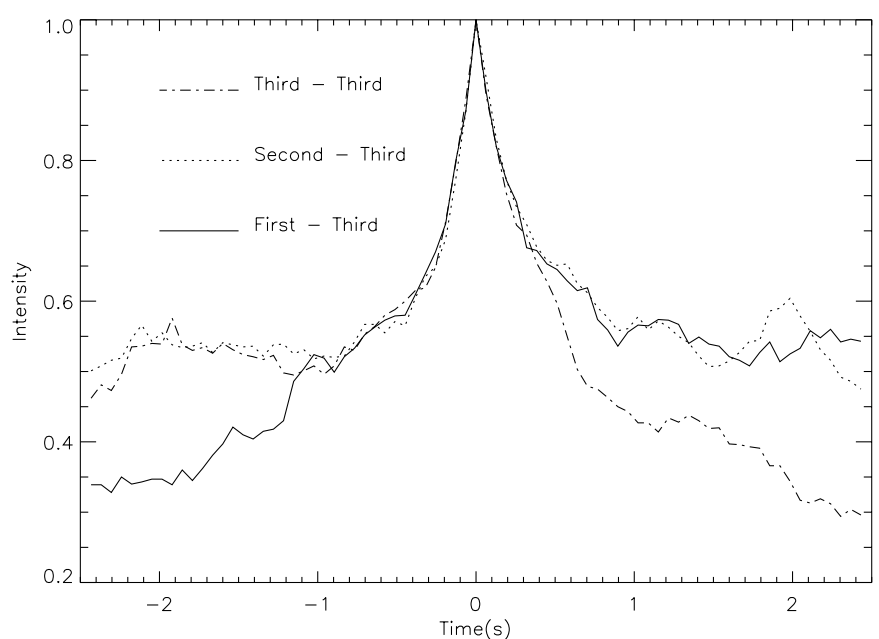

Fig. 1. Average peak alignment from 53 bright BATSE bursts with durations longer than $20 \mathrm{~s}$. The three curves show the average pulse shape for the largest peak in the first third, second third, and last third of the bursts. We find no significant change, during the gamma-ray phase, in the average peak width over at least $2 / 3$ of $T_{90}$. Models should account this empirical trend in GRB physics

We have shown the lack of temporal evolution of the peak width in the context of an average of many bursts. Now, we expand our analysis to individual bursts. An excellent analysis has been provided by Norris et al. (1995), where they examined the temporal profiles of bright GRBs by fitting those profiles with pulses. From the set of bursts that they analysed, we used the 28 bursts with the characteristic of having seven or more fitted pulses within their duration. To obtain the temporal dependency of the pulse width, we selected the five largest peaks in each burst and fit their FWHM to a function of the arrival time, $\Delta T=k\left(\frac{T-T_{\mathrm{c}}}{T_{90}}\right)^{\alpha}$. We chose this model because the expected dependency from the "external" shock model scales as $\frac{T / T_{90}}{\Gamma(1+\beta)}$ (if a relativistic shell is responsible for the shape in the time histories, $T_{0}$ should be proportional to $T_{90}$; see Fenimore et al. 1996). The purpose of the $T_{\mathrm{c}}$ parameter is to correct the BATSE time to the time since the beginning of the explosion. Figure 2 shows the distribution of the power law indexes $(\alpha)$ for the set of bursts analysed. The distribution shows that in individual bursts the pulse width does not increase throughout their duration as predicted by the external shock model, for which $\alpha$ is expected to be 1 (or larger if deceleration is occurring). In fact, most bursts show a diminution in pulse width.

\section{Discussion}

We have uncovered that the width of the peaks remains remarkably constant throughout a burst. The width should scales as $\Gamma(1-\beta \cos \theta)$. In the external shock model, such lack of temporal evolution implies that $\Gamma$ must be nearly constant. Peaks occur late in the burst because they are

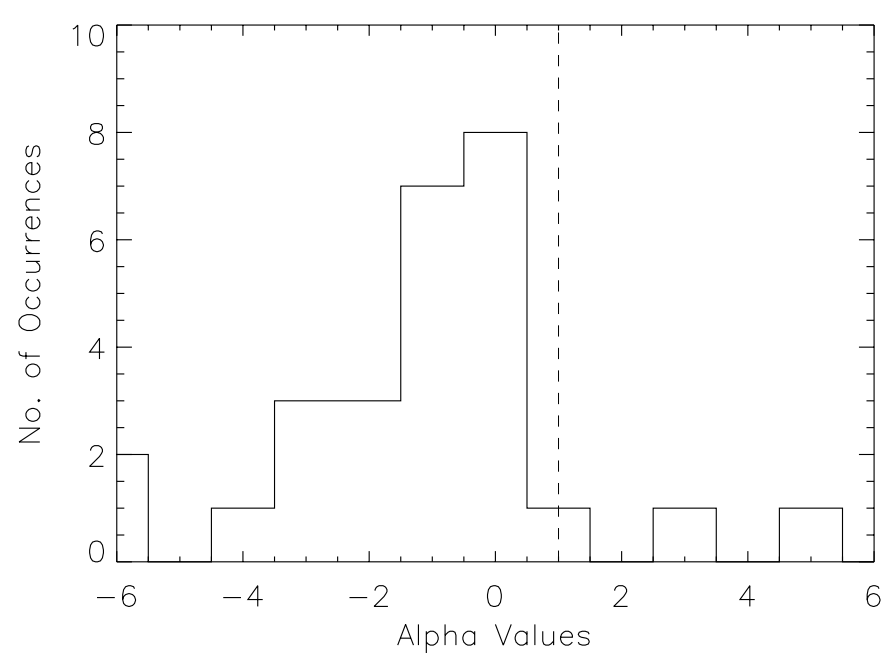

Fig. 2. Distribution of the $\alpha$ parameter from 28 bright BATSE bursts with durations longer than $1.5 \mathrm{~s}$. The pulse width $(\Delta T)$, as expected for the external model, should scale as $T / T_{90}$. We fit $k\left(\frac{T-T_{\mathrm{c}}}{T_{90}}\right)^{\alpha}$ to find the temporal evolution of the pulse width in each burst. The dashed line is the expected index value, $\alpha$, for a single relativistic shell $(\alpha=1)$. The distribution shows that the vast majority of the bursts present no time evolution (or negative) of the pulse width

from regions off axis where the delay is caused by the curvature of the shell. The later peaks would be wider and delayed because off axis regions have larger $\theta$ 's. A constant peak width indicates that all emitting entities must have similar $\theta$. Since the maximum angular size of the shell allowed by a differential spread of $S$ is $S \Gamma^{-1}$, the entire size of the shell must be a few percent of $\Gamma^{-1}$. Thus, the only external shock model that is consistent with the observations is one where the overall size of the shell is much smaller than $\Gamma^{-1}$ and there is no deceleration during the classic GRB phase. This adds to our previous arguments (Fenimore et al. 1996; Fenimore et al. 1998) that a central engine (internal shocks) is the more likely explanation for the observed chaotic time history. In the context of the internal shock model, we cannot place a limit on angular extent but the lack of evolution of the peak width indicates that $\Gamma$ must be remarkably constant throught the internal shock phase.

\section{References}

Fenimore E.E., Madras C.D., Nayakshin S., 1996, ApJ 473, 998, astro-ph/9607163

Fenimore E.E., Ramirez-Ruiz E., Sumner M.C., 1998, GammaRay Bursts: 4th Huntsville Symposium, Meegan, Preece and Koshut (eds.), (AIP Proc. 428), astro-ph/9712303, p. 657

Li H., Fenimore E.E., 1996, ApJ 469, L115

Mitrofanov I.G., 1997, ApJ 490, 509

Norris J.P., et al., 1996, ApJ 459, 393

Sari R., Piran T., 1997, ApJ 485, 270 